\title{
Structure and control of self-sustained target waves in excitable small-world networks
}

\author{
Yu Qian, ${ }^{1,2}$ Xiaodong Huang, ${ }^{1}$ Gang $\mathrm{Hu}, 1, * *$ and Xuhong Liao ${ }^{1,+}$ \\ ${ }^{1}$ Department of Physics, Beijing Normal University, Beijing 100875, China \\ ${ }^{2}$ Nonlinear Research Institute, Baoji University of Arts and Sciences, Baoji 721007, China
}

(Dated: November 5, 2018)

\begin{abstract}
Small-world networks describe many important practical systems among which neural networks consisting of excitable nodes are the most typical ones. In this paper we study self-sustained oscillations of target waves in excitable small-world networks. A novel dominant phase-advanced driving (DPAD) method, which is generally applicable for analyzing all oscillatory complex networks consisting of nonoscillatory nodes, is proposed to reveal the self-organized structures supporting this type of oscillations. The DPAD method explicitly explores the oscillation sources and wave propagation paths of the systems, which are otherwise deeply hidden in the complicated patterns of randomly distributed target groups. Based on the understanding of the self-organized structure, the oscillatory patterns can be controlled with extremely high efficiency.

PACS numbers: : 82.40.Ck, 05.65.+b, 87.18.Bb, 89.75.Hc
\end{abstract}

\section{INTRODUCTION}

Pattern dynamics in excitable media has attracted great attention in wide fields due to its relevance to various important systems, such as cardiac tissues and neural systems for typical examples [1-3]. Though single excitable cell is not oscillatory, organized oscillatory patterns, however, are extremely important issues in the media of coupled excitable cells [4-6]. Spiral waves and target waves are two typical patterns in excitable tissues [1, 7]. The former can self-sustain in autonomous systems with the spiral tips serving as the oscillation sources while the latter can exist only by external pacing and can never exist in autonomous regular excitable media. So far most of researches studying pattern dynamics of excitable tissues have focused on locally and regularly coupled media, and little has been known on the influences of long-range or random links on the system dynamics. In many realistic systems of great importance, of which neural networks are typical examples, these long-range couplings do exist and they play crucial roles in deciding the functions of the systems [8-15]. Recently, A. Roxin et al. [16], S. Sinha et al. [17], A. J. Steele et al. [18] and Gray et al. [19] studied excitable systems in small-world networks and found some new types of self-sustained oscillations, including target waves in autonomous systems[18,19]. However, the

\footnotetext{
*Electronic address: ganghu@ bnu.edu.cn

†Electronic address: liaoxuhong@mail.bnu.edu.cn
} 
mechanism underlying these new types of oscillations and the effective methods to control and regulate the oscillatory dynamics have far from been clear.

In this paper we study the oscillatory behaviors of self-sustained target waves in small-world networks of excitable nodes. We proposed a novel method of dominant phase-advanced driving to reveal the successive driving structures supporting the self-sustained oscillations, based on the known network structure and oscillation data. In these structures oscillation sources and wave propagation pathways are explored explicitly, which are otherwise deeply hidden in randomly distributed target groups. Based on the structure we are able to effectively control and regulate the oscillations of the excitable networks by suitably manipulating very few long-range links.

The paper is arranged as follows. In Sec. II we describe the basic idea and the operating procedure of dominant phase-advanced driving (DPAD) method. Then we explain based on the graph theory why this method can reveal the essential structure generating self-sustained oscillations from complicated oscillatory patterns of complex networks. In Sec. III we study a model of small-world networks consisting of excitable nodes. We reveal both oscillatory patterns of spiral waves and multiple-target waves. The mechanism of the former case is well known - spiral wave tips serve as the oscillation generators. In the latter case, we apply the DPAD method and explicitly explore the wave source and the wave propagation pathways. In Sec. IV we show how to effectively control self-sustained target patterns based on the above understanding. In Sec. V we analyze the conditions for the applications of the DPAD method, and show how this method can provide useful understanding even if partial necessary information are not available. The last section gives brief discussion on the results and the significance of our method.

\section{ANALYZING SELF-SUSTAINED OSCILLATION OF COMPLEX NETWORKS BY USING DPAD METHOD}

Considering a general network graph $G(V, E)$ with $V$ representing a set of $N$ nodes and $E$ being a set of $M$ interactions (i.e., couplings). Dynamic variables are associated to each node, and these variables obey well defined coupled ordinary differential equations (ODEs). Each node is nonoscillatory individually while the entire complex networks are periodically oscillatory. It is well known that a necessary condition for this type of oscillatory networks is that there must exist some interacting loops. For any connected network with $N$ nodes and $M$ interactions, the number of fundamental cycles is $M-N+1$ [20]. And the number of topological cycles serving as the candidates of source loops are $2^{M-N+1}-1$, which is huge in the case $M \gg N$ (this is so for most of practical networks). Cycles are referred to as loops in our paper for simplicity. In this section we will propose an effective method to identify the key dynamic loops generating the oscillations from the large number of topological loops. 
Regardless of different dynamics and different coupling forms, we propose a common design principle for such oscillatory networks.

Design principle: each nonoscillatory node can oscillate if and only if it is driven by one or few oscillatory interactions with advanced phases.

The definitions of "advanced phase"for different systems can be different, but they are the same in essence[21]. Let us consider an example of simplest $1 D$ oscillatory networks consisting of nonoscillatory nodes where each node is unidirectionally interacted by only a single other node as shown in Fig. 1, The network consists of $N$ nodes with $M(M=N)$ unidirectional interactions. As the network is oscillatory, all the noes are activated. Suppose an arbitrary node $i_{1}$ is phase-advancedly driven by a node $i_{2}$ via coupling, which is phase-advancedly driven by node $i_{3}$ in turn, and this successive unidirectional driving chain goes as $i_{1} \leftarrow i_{2} \leftarrow i_{3} \leftarrow \cdots \leftarrow i_{k} \leftarrow \cdots$. Since $N$ is finite we must come to a node $i_{q}, q \leq N$, which is driven by one of the previous nodes $\left\{i_{1}, i_{2}, \cdots, i_{q-1}\right\}$, said $i_{p}(p<q)$. Then a successive regulatory loop $i_{p} \leftarrow i_{p+1} \leftarrow \cdots \leftarrow i_{q} \leftarrow i_{p}$ is formed, serving as the oscillation source of all other nodes. From the graph theory, this network has one and only one fundamental loop $(N-N+1=1)$, and this fundamental loop is right the dynamic driving loop playing the role of the oscillation source, while all other nodes must in the tree branches radiating from this loop identifying wave propagation paths. The simple structure in Fig. 1 consists of trees from loops, thus is called trees from loops (TFL) pattern.

The TFL structure of Fig. 1 is universal for all self-sustained periodic oscillations in complex networks consisting of nonoscillatory nodes. It illustrates the simplest relationship of these nodes in self-sustained oscillation. Since no nonoscillatory node can oscillate without phase-advanced driving from other nodes, two key rules must be obeyed by any TFL structure:

(i) There must be some (at least one) successively phase-advanced driving loops.

(ii) Each node not in the loops must be in a tree branch rooted at a node in a loop.

The simple and instructive structure of Fig. 1 gives an example of simplest $1 D$ network which can selfsustainedly oscillate. However, interaction structures of complex networks are in general much more complex than Fig. 1 which are high dimensional and random (e.g. Fig. 2(a)). In Fig. 1 the only topological loop is right the source loop generating the oscillation. In practical complex connected networks we usually have $M \gg N$, and the number of topological loops is large. We therefore propose an operable and physically meaningful method to reduce the original random network graphs (as Fig. 2(a)) to the simple and instructive TFL patterns of Fig. 1, The method consists of the following Complexity Reduction steps:

(a) Find phase-advanced driving interactions for each node.

(b) Find the single dominant interaction among these phase-advanced driving interactions. 
(c) Use all these dominant interactions to unidirectionally link the network nodes, and draw the dominant phase-advanced path pattern, which represents the dynamic geodesic of the complex network, showing the most significant (or say the shortest) driving paths in the given oscillatory states. This pattern reduce the original network $G(V, E)$ to a new graph $G\left(V, E^{\prime}\right)$ with $E^{\prime}$ being a subset of $E\left(E^{\prime} \in E\right)$.

Note, we have $M^{\prime}=N$ since each node must have one and only one dominant phase-advanced driving, and thus this graph is nothing but the $1 D$ TFL pattern of Fig. 1, where the loop is the core topology for the oscillation serving as the source loop and the unidirectional links indicate the wave propagation pathways.

All the above steps rely on only the necessary regulating topology under the general condition: any nonoscillatory node can oscillate only if it is driven by phase-advanced interactions from other nodes. They are widely applicable in diverse fields for self-sustained oscillations of complex networks of individually nonoscillatory nodes, independing of node dynamics (excitable or nonexcitable), coupling forms (active interactions or repressive interactions, directional or symmetric couplings), and network structures (smallworld networks, purely random networks, or scale free networks). The particular meanings of "advanced phase" and "dominant phase-advanced driving" should be properly defined, according to realistic physical, chemical and biological interaction mechanisms in each individual system. In the following sections we will apply this method to particular systems of oscillatory excitable small-world networks.

\section{SELF-SUSTAINED TARGET WAVES IN EXCITABLE SMALL-WORLD NETWORKS}

We take a two-dimensional (2D) Bär model [22] as our example

$$
\begin{gathered}
\dot{u}_{i, j}=-\frac{1}{\varepsilon} u_{i, j}\left(u_{i, j}-1\right)\left(u_{i, j}-\frac{v_{i, j}+b}{a}\right)+D_{i, j}, \\
\dot{v}_{i, j}=f\left(u_{i, j}\right)-v_{i, j}, \\
D_{i, j}=D_{u}\left(u_{i-1, j}+u_{i+1, j}+u_{i, j-1}+u_{i, j+1}-4 u_{i, j}\right) .
\end{gathered}
$$

where $f\left(u_{i, j}\right)=0$ for $u_{i, j}<\frac{1}{3} ; f\left(u_{i, j}\right)=1-6.75 u_{i, j}\left(u_{i, j}-1\right)^{2}$ for $\frac{1}{3} \leq u_{i, j} \leq 1$; and $f\left(u_{i, j}\right)=1$ for $u_{i, j}>1$. The system parameters are kept throughout this paper as $a=0.84, b=0.07, \varepsilon=0.04$ and $D_{u}=1.0$ just for the sake that the local cell follows excitable dynamics. In the present paper we consider $100 \times 100$ cells in the $2 D$ regular lattice with constant and homogeneous nearest couplings. With this coupling topology, spiral waves can be easily observed for random initial conditions. It is well known (and also it is verified in our simulations) that target waves can never be observed in the asymptotic states of Eq. (1) unless some persistent external pacings provide the wave sources. Many systems of practical importance, such as neural 
systems, have complex coupling structures where cells can be coupled to each other with both short-range and long-range couplings. For studying the influence of long-range couplings (L-RCs) we add an additional coupling term $D_{i, j}^{\prime}$ to Eq. (1a) as

$$
\begin{gathered}
\dot{u}_{i, j}=-\frac{1}{\varepsilon} u_{i, j}\left(u_{i, j}-1\right)\left(u_{i, j}-\frac{v_{i, j}+b}{a}\right)+D_{i, j}+D_{i, j}^{\prime}, \\
\dot{v}_{i, j}=f\left(u_{i, j}\right)-v_{i, j}, \\
D_{i, j}^{\prime}=\left\{\begin{array}{cc}
D_{u}\left(u_{i^{\prime}, j^{\prime}}-u_{i, j}\right) & \text { for }\left(i, j ; i^{\prime}, j^{\prime}\right) \in \Omega, \\
0, & \text { otherwise, }
\end{array}\right.
\end{gathered}
$$

where $\Omega$ is a set of $K \mathrm{~L}-\mathrm{RCs}$ between non-neighbor sites $(i, j)$ and $\left(i^{\prime}, j^{\prime}\right)$ randomly chosen in the $2 D$ lattice. Eq. (2) is integrated by the second-order Runge-Kutta scheme with the time step $\Delta t=0.031$ and the No-Flux boundary condition is used.

Now we consider a small-world network of Eq. (2) with $K=150$ random L-RCs which is shown in Fig. 2(a). We run the system from 100 sets of random initial conditions and find 3 realizations for homogeneous rest state; 63 for various spiral wave patterns (one of them is shown in Fig. 2 (b)); and 34 for self-sustained target wave patterns (two of them are shown in Figs. 2(c) and 2(d)). The mechanism of oscillation of the spiral wave of Fig. 2(b) is well understood: the spiral tip plays the role of oscillation source and waves propagate from the tip to far away. However, from the randomly distributed groups of target waves of Figs. 2(c) and 2(d) we can hardly say anything about the mechanism underlying different patterns, for instance, where the oscillation sources are and how waves propagate from the sources to the whole tissue. Specifically, in both patterns there are large numbers of target centers, we may ask which centers are the true centers (sources) of the oscillations.

We apply the DPAD method to analyze the oscillation patterns of Figs. 2(c) and 2(d). First we should specify the definitions of phase-advanced and dominant phase-advanced driving for the sepcific systems of Eq. (2). For a given ith cell, we define the phase-advanced driving as the interactions from all the neighbors which have $u(t)>u_{i}(t)$ at time $t_{e}$ when the $i t h$ cell is kicked from the rest state (i.e., $u_{i}(t)$ crosses the threshold value $u_{e}=\frac{b}{a}$ from small value). Among all these phase-advanced interactions we define the interaction from the node, which has the largest $u(t)$ at $t_{e}$, as the dominant driving. Therefore, each cell is associated with only a single DPAD. These definitions are clearly demonstrated in Fig. 3 (a) by studying a node 8250 of Fig. 2(c) as an example where node 8249 provides the dominant phase-advanced driving to the given node 8250 , and we can draw an arrowed driving path from 8249 to 8250 in the corresponding TFL pattern of Fig. 3(b). 
Now we use this definition to draw the TFL patterns based on known interaction structure and oscillation data. Figures. 3(b) and 3 (c) show TFL structures corresponding to the oscillation patterns of Figs. 2(c) and 2(d), respectively. From the TFL patterns all the above questions can be understood without any ambiguity. First, we have revealed in Fig. 3(b) and Fig. 3)(c) the wave sources - 1D source loops (linked by the pink nodes) from the large number of possible candidates of topological loops in Fig. 2(a). In the source loops all red bold arrowed lines show L-RCs $\left(A_{1}^{\prime} \longrightarrow B_{1}, B_{1}^{\prime} \longrightarrow A_{1}\right.$ in Fig. 3 (b); $A_{2}^{\prime} \longrightarrow B_{2}, B_{2}^{\prime} \longrightarrow C_{2}, C_{2}^{\prime} \longrightarrow A_{2}$ in Fig. 3(c)) and all other arrowed lines come from local chains. In Figs. 2(c) and 2(d) all the local chains in the source loops are shown by bold lines, and the source centers in each pattern are identified by red disks, such as: double centers $\left(A_{1}, B_{1}\right)$ with the source loop $A_{1} \stackrel{\text { bold line }}{\longrightarrow} A_{1}^{\prime} \stackrel{L-R C}{\longrightarrow} B_{1} \stackrel{\text { bold line }}{\longrightarrow} B_{1}^{\prime} \stackrel{L-R C}{\longrightarrow} A_{1}$ for Fig. 2(c); and triple centers $\left(A_{2}, B_{2}, C_{2}\right)$ with the source loop $A_{2} \stackrel{\text { bold line }}{\longrightarrow} A_{2}^{\prime} \stackrel{L-R C}{\longrightarrow} B_{2} \stackrel{\text { bold line }}{\longrightarrow} B_{2}^{\prime} \stackrel{L-R C}{\longrightarrow}$ $C_{2} \stackrel{\text { bold line }}{\longrightarrow} C_{2}^{\prime} \stackrel{L-R C}{\longrightarrow} A_{2}$ for Fig. 2(d).

Via TFL patterns of Figs. 3(b) and3(c) we can not only understand the problem which target centers are the true centers of the oscillations (red square nodes), but also understand how waves propagate from the source centers to all the sub-target centers ( $S T C s$, shown by blue square nodes), and then produce groups of target waves successively in the patterns. Taking the sub-target center $S T C_{6}$ in Fig. 2 (c) as an example, we realize from the TFL pattern of Fig. $3(\mathrm{~b})$ that waves start from the source target center $A_{1}$ and propagate through the path $A_{1} \longrightarrow S T C_{4} \longrightarrow S T C_{6}$. All these sub-target centers take their fixed positions in the TFL structures, and the problems how these centers are driven by upstream nodes (through L-RCs) and how they drive their downstream nodes (through various sub-target waves supported by local couplings) are illustrated clearly.

\section{CONTROL OF SELF-SUSTAINED OSCILLATIONS IN EXCITABLE COMPLEX NETWORKS}

The most interesting point is that we can perform pattern control and regulation based on the TFL patterns. Our task is to effectively suppress oscillations. By "effective" we mean to change as small as possible number of couplings. If TFL patterns in Fig. 3 make sense we expect that removing a single L-RC on the source loop can suppress the given target waves in the whole pattern. In Fig. 4(a) we plot $<u(t)>=\frac{1}{N^{2}} \sum_{i, j=1}^{N=100} u_{i, j}(t)$ and $<v(t)>=\frac{1}{N^{2}} \sum_{i, j=1}^{N=100} v_{i, j}(t)$ vs $t$, and show that $<u(t)>$ and $<v(t)>$ damps to zero after a single L-RC $B_{1}^{\prime} A_{1}$ of Fig. $2(\mathrm{c})$ is discarded. A snapshot of pattern evolution after discarding $B_{1}^{\prime} A_{1}$ is presented in Fig. 4(b) where the system is approaching to the homogeneous rest state. The damping process after discarding L-RC $B_{1}^{\prime} A_{1}$ can be well explained based on the TFL pattern of Fig. 3 (b). When we remove $B_{1}^{\prime} A_{1}$, the source loop $A_{1} \longrightarrow A_{1}^{\prime} \longrightarrow B_{1} \longrightarrow B_{1}^{\prime} \longrightarrow A_{1}$ breaks, and thus the source centers $A_{1}$, $B_{1}$ no longer emit waves, and the target waves from centers $A_{1}, B_{1}$ first damp. Without excitations from 
these sources, all other target centers cease to emit waves successively due to the successive annihilations of their driving waves. Finally, the whole pattern with all waves generated by a large numbers of target centers evolves to the homogeneous rest state. Similar control effects are observed for other self-sustained target patterns. When any single long-range link in the source loop is discarded, the given oscillation of the whole system is destroyed completely, and the system either evolves to the homogeneous rest state, or develops into an entirely different oscillatory structure due to the reconstruction of a different new source loop. It is really striking that by discarding only a single L-RC link among $2 \times 10^{4}$ local couplings and 150 L-RCs we can essentially change the dynamics of the whole pattern formation. This can never happen for spiral waves.

It is emphasized that the self-sustained target waves are robust against random changes of the coupling structure. For instance, the main structure of pattern Fig. 2(c) is not considerably changed if we discard all L-RCs except the 9 relevant L-RCs shown in Fig. 2(c). A snapshot of pattern after discarding such 141 L-RCs are shown in Fig. 4(c). Fig. 4(d) shows the asymptotic pattern when we discard all 148 L-RCs in Fig. 2(c) except the 2 L-RCs $A_{1}^{\prime} B_{1}$ and $B_{1}^{\prime} A_{1}$ in the source loop. The oscillation continues just with these two long-range links. The comparison of Fig. 4(d) (discarding 148 L-RCs and keeping only the two L-RCs in the source loop) with Fig. 4(b) (discarding only a single L-RC in the source loop) is really striking.

\section{ROBUSTNESS OF APPLICATIONS OF THE DPAD METHOD}

In the above applications of the DPAD method, we required full and precise knowledge on the interaction structures of networks and the variable data of oscillatory nodes. In realistic cases, the information is often not complete, it is thus necessary to consider the robustness of our approach in these practical situations.

First, in practical experiments the measured data are often not precise, i.e., we should consider noisy data. In these cases, our method has an advantage of robustness. The validity of the DPAD method does not rely on precise values of data measurements, but depend on the topological orders of data, such as phase-advanced or phase-delayed interactions and dominant or nondominant drivings. These topological orders can often be correctly explored when the data measurements have certain small errors. Moreover, for periodic oscillations we may reduce some random measurement errors by averaging the data in sufficiently long intervals by making optimal tradeoff between the precision and the measurement expense. Those treatments are applicable when the errors are relative small. If the errors are large, the methods may no longer work.

Now we focus on the second and the most important problem: the robustness of the DPAD method against the incompleteness of information. Another promising advantage of our method is that the pathways of TFL 
patterns are drawn based on local knowledge, i.e., the driving path of a given node is determined by the oscillation data of this node and few nodes in direct interactions. Lack of information around certain node does not affect the applications of the DPAD method to nodes far away. In these cases it is possible to draw incomplete TFL patterns from incomplete knowledge, and these unperfect TFL patterns may still provide useful and instructive guidance in analyzing the oscillations. Here we only consider a relatively simple situation of information lacks - incomplete interaction structure.

We again consider the state of Fig. 2(c), and apply the DPAD method with incomplete interaction knowledge. Specifically, we draw the TFL pattern under the condition that $50 \mathrm{~L}-\mathrm{RC}$ randomly chosen unknown. One of these TFL patterns is shown in Fig. 5(a). Comparing Fig. 5(a) with Fig. 3(b) we find while Fig. 3(b) has a single connected network, Fig. 5(a) has at most 51 clusters disconnected from each other (only 4 clusters are shown in Fig. 5(a)). In the incomplete TFL pattern we observe some tree-like networks each having a source node controlling the downstream nodes. We also find a cluster having a TFL loop which is exactly the same as the source loop of Fig. 3(b). It is obvious that Fig. 5(a) keeps the essential structure of the complete TFL shown in Fig. 3 (b). In particular, from Fig. 5(a) we see clearly the oscillation source and we can locate the two key L-RCs in the source loop which control the whole oscillatory state. In Fig. 5(b) we present another TFL pattern for a different set of unknown 50 long-range interactions, where a L-RC on the source loop of Fig. 3(b) is, unfortunately, chosen to be unknown. Now we observe no loop but at most 50 tree clusters (4 clusters shown in Fig. 5(b)), each is driven by a source node. And the source nodes themselves are not driven by any other node in the incomplete TFL. It becomes difficult to locate the source loop without some additional information. Nevertheless, the incomplete TFL pattern of Fig. 5(b) can still show rich driving paths of the state Fig. 2 (c) and it may serve as an excellent guidance for analyzing the oscillation organization. The following understanding is of great help in estimating the unknown L-RCs of the source nodes. (i) Each source node must be driven by a L-RC phase-advanced. (ii) In TFL pattern the phase differences between all pairs of target centers and their corresponding upstream driving nodes are approximately the same (they are about $1.91 \mathrm{rad}$ in our system as shown in Fig. 5(c)). This phase difference can be used for seeking the upstream driving nodes of the unknown L-RCs. (iii) The "source" node controlling the largest tree cluster of Fig. 5(b) has large probability to be a key node in the source loop (this conclusion is confirmed by all 10 test, using different sets of unknown L-RCs). These conclusions, which may be popular for self-sustained target waves in small-world excitable networks, can considerably reduce the difficulty in recovering the complete TFL. If we have some additional (still not complete) information which may be model dependent, the above conclusions may be used for predicting the missing long-range driving links of the source nodes and recovering the complete TFL structure. For instance, in our system 
all the periodic orbits of upstream driving nodes of L-RCs have similar characteristic which is considerably different from normal nodes (see Fig. 5 (c) for an example). Jointly using this particular feature and the above three general conclusions we can explore the missing L-RC drivings of source loop and all other missing L-RCs of source nodes with extremely high probability. For instance, in 10 random samples we successfully predict all the missing L-RCs of source nodes and recover the original main TFL structure without any error, of which the patching from Fig.5(b) is shown in Fig. 5(d).

Furthermore, we have tested many other cases with different system sizes, different numbers of existing L-RCs and different unknown L-RCs. We found that the above method of exploring unknown L-RCs works fairly well, and we can correctly reveal TFL loops with high probability. However, the method is not always successful. It may fail when different source nodes are linked by L-RCs having similar time series. The recover operations will be even more difficult when both node data and interaction structure are incomplete. We will go further into these cases in our future study.

\section{CONCLUSION}

In conclusion we have studied periodic self-sustained oscillations of target waves in excitable small-world networks. Based on interaction structures and oscillation data, a novel method of dominant phase-advanced driving (DPAD) path is proposed to study self-sustained oscillations in complex networks of nonoscillatory nodes and tree from loop (TFL) patterns can be drawn for oscillatory states. For oscillatory excitable smallworld networks these TFL patterns show clearly 1D loops serving as oscillation sources and illustrate how waves propagate from the source loops to the whole tissues via various local and long-range couplings of small-world networks. More interestingly, with the instructive information of TFL patterns we can control and regulate oscillatory patterns of small-world networks with extremely high efficiency. Self-sustainedly oscillatory complex networks of excitable cells exist significantly in wide fields, such as neural systems. Both the DPAD method and TFL structures are thus expected to be of broad interest in crossing fields of nonlinear dynamics, complex networks and their practical applications. In the present paper we consider only periodic oscillations of fixed complex networks. The extensions to nonperiodic oscillations (e.g., chaotic) and to the cases of noise and parameter-variation-driven oscillations, which occur popularly in practical situations, are future interesting tasks. 


\section{Acknowledgments}

This work was supported by the National Natural Science Foundation of China under Grant No. 10675020 and by the National Basic Research Program of China (973 Program) under Grant No. 2007 CB814800. 
[1] A. T. Winfree, When Time Breaks Down (Princeton University Press, Princeton, NJ, 1987).

[2] R. A. Gray, A. M. Pertsov, and J. Jalife, Nature (London) 392, 75 (1998).

[3] T. P. Vogels, K. Rajan, and L. F. Abbott, Annu, Neurosci 28, 357 (2005).

[4] M. Bazhenov, I. Timofeev, M.steriade, and T. J. Sejnowski, Nature Neuroscience 2, 168 (1999).

[5] M. Steriade and I. Timofeev, Neuron 37, 563 (2003).

[6] G. Buzsaki and A. Draguhn, Science 304, 1926 (2004).

[7] A. Mikhailov, Foundations of Synergetics (Springer-Verlag, Berlin, 1994).

[8] D. J. Watts and S. H. Strogatz, Nature (London) 393, 440 (1998).

[9] A.-L. Barabasi and R. Albert, Science 286, 509 (1999).

[10] V. M. Eguiluz, D. R. Chialvo, G. A. Cecchi, M. Baliki, and A. V. Apkarian, Phys. Rev. Lett. 94, 018102 (2005).

[11] F. Qi, Z. Hou, and H. Xin, Phys. Rev. Lett. 91, 064102 (2003).

[12] A. Zumdieck, M. Timme, T. Geisel, and F. Wolf, Phys. Rev. Lett. 93, 244103 (2004).

[13] L. F. Lago-Fernandez, R. Huerta, F. Corbacho, and J. A. Siguenza, Phys. Rev. Lett. 84, 2758 (2000).

[14] D. He, G. Hu, M. Zhan, W. Ren, and Z. Gao, Phys. Rev. E 65, R055204 (2002).

[15] S. Yonker and R. Wackerbauer, Phys. Rev. E 73, 055206 (2006).

[16] A. Roxin, H. Riecke, , and S. A. Solla, Phys. Rev. Lett. 92, 198101 (2004).

[17] S. Sinha, J. Saramäki, and K. Kaski, Phys. Rev. E 76, R015101 (2007).

[18] A. J. Steele, M. Tinsley, and K. Showalter, Chaos 16, 015110 (2006).

[19] R. A. Gray, A. M. Pertsov, and J. Jalife, Circulation 94, 2649 (1996).

[20] B. Bollobás, Modern Graph Theory (Springer-Verlag, New York, 1998).

[21] For a given node (say, node A), we define all interactions favorable to activating node A from the rest state as phase-advanced interactions, and among the phase-advanced interactions we define the interaction providing the most significant activating contribution as the dominant phase-advanced driving (DPAD).

[22] M. Bár and M. Eiswirth, Phys. Rev. E 48, R1635 (1993). 


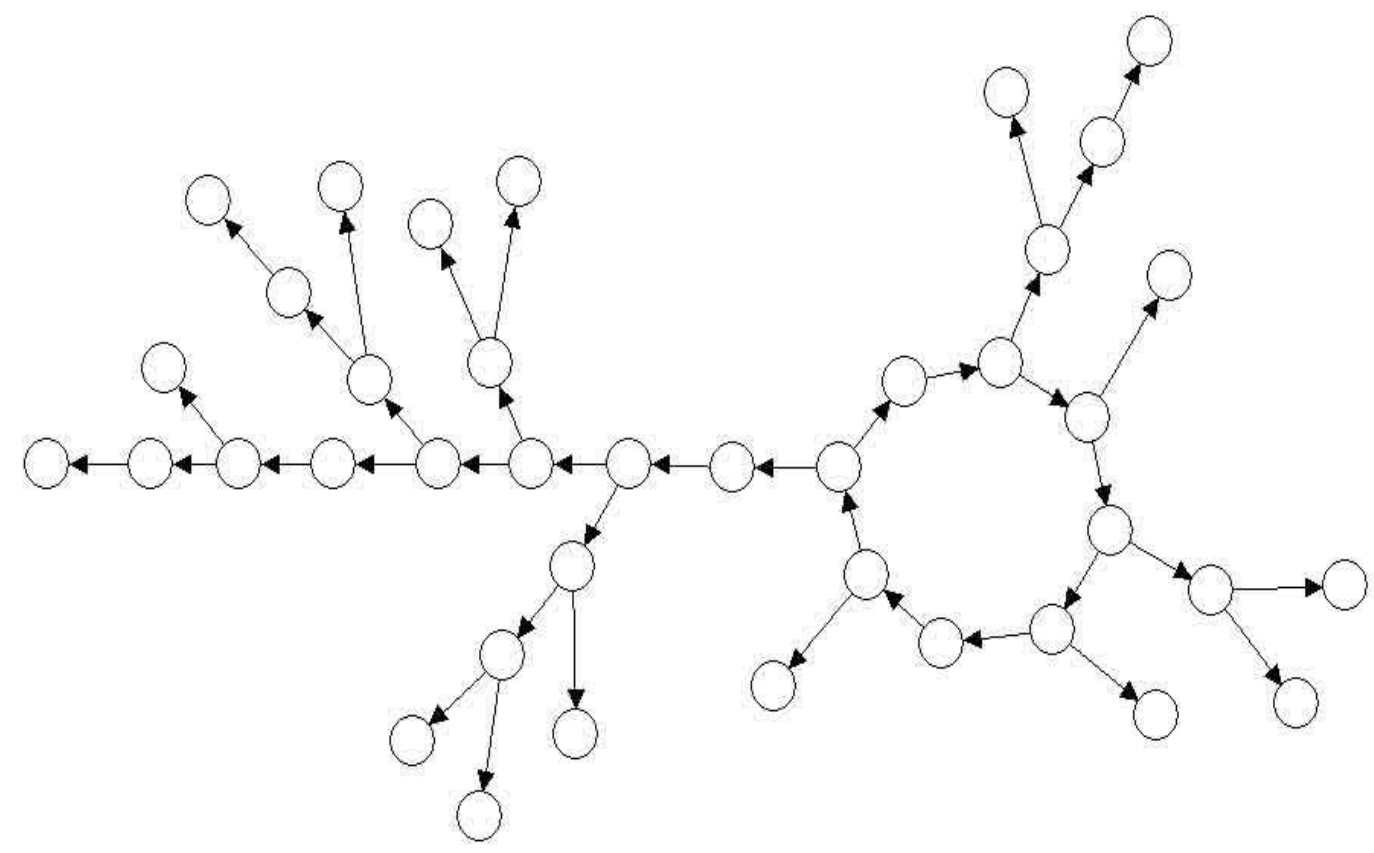

FIG. 1: Schematic figure of universal structure of periodically oscillatory 1D network consisting of nonoscillatory nodes. The figure has a form of tree branches radiating from an interacting loop, and is called tree from loop (TFL) structure. 


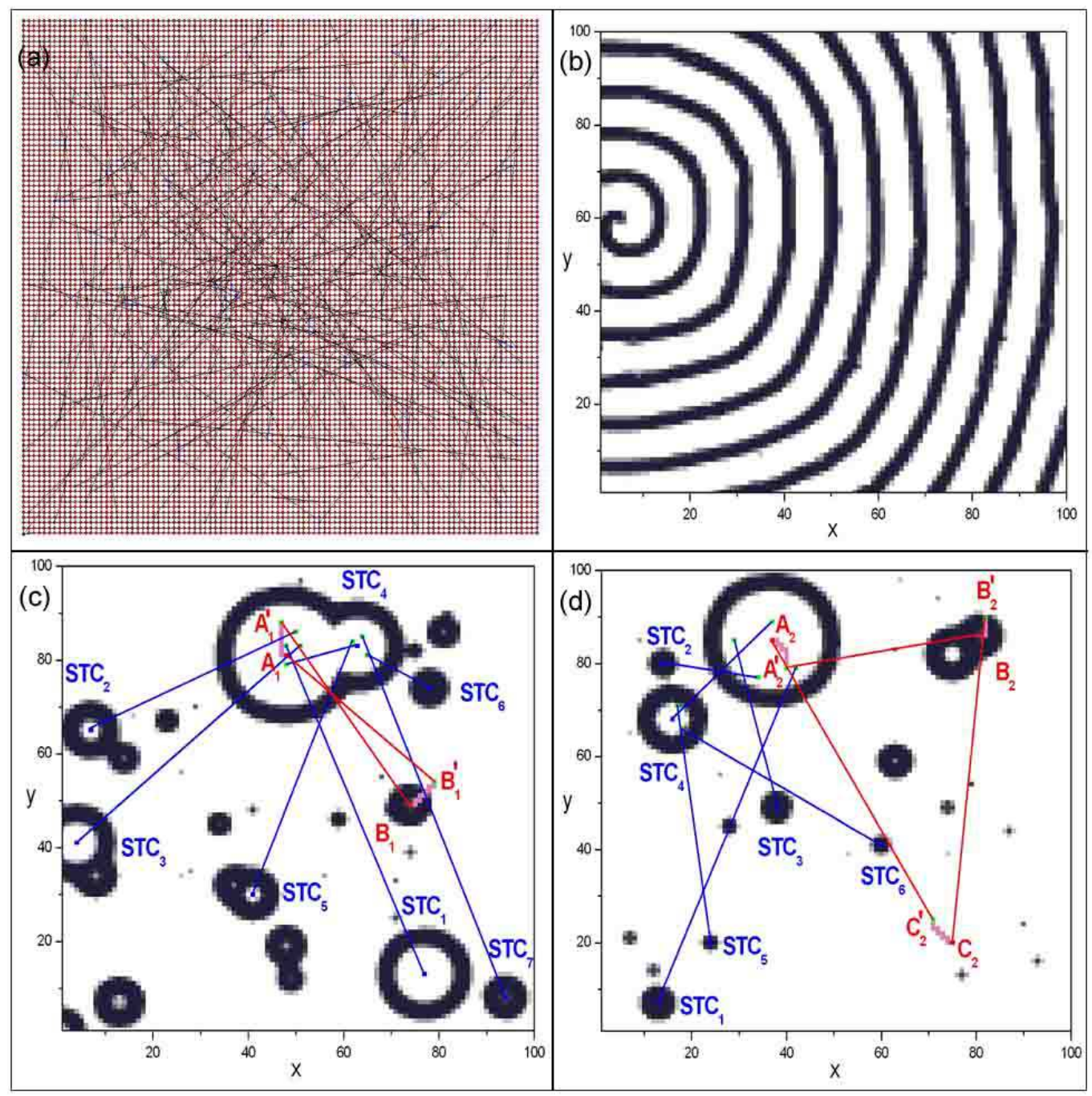

FIG. 2: Coloured online. (a) A small-world network with 150 random long-range links (note, the tissue has about $2 \times 10^{4}$ nearest-neighbor links). (b) Spiral waves realized from a set of initial condition. (c) (d) Different asymptotic target wave patterns from two different initial conditions. The red nodes $\left(A_{1}, B_{1}\right)$ and $\left(A_{2}, B_{2}, C_{2}\right)$ denote the centers of the target waves located in the source loops of Fig. 3 called source centers, and the blue nodes $S T C_{i}(i=1,2, \cdots$,$) denote the sub-target centers driven by the source targets. All the green nodes$ denote the nodes connected with the various target centers with long-range interactions. The source loops found in Fig. 3 are denoted here $A_{1} \stackrel{\text { bold line }}{\longrightarrow} A_{1}^{\prime} \stackrel{L-R C}{\longrightarrow} B_{1} \stackrel{\text { bold line }}{\longrightarrow} B_{1}^{\prime} \stackrel{L-R C}{\longrightarrow} A_{1}$ in (c); and $A_{2} \stackrel{\text { bold line }}{\longrightarrow} A_{2}^{\prime} \stackrel{L-R C}{\longrightarrow} B_{2} \stackrel{\text { bold line }}{\longrightarrow} B_{2}^{\prime} \stackrel{L-R C}{\longrightarrow} C_{2} \stackrel{\text { bold line }}{\longrightarrow} C_{2}^{\prime} \stackrel{L-R C}{\longrightarrow} A_{2}$ in (d). 


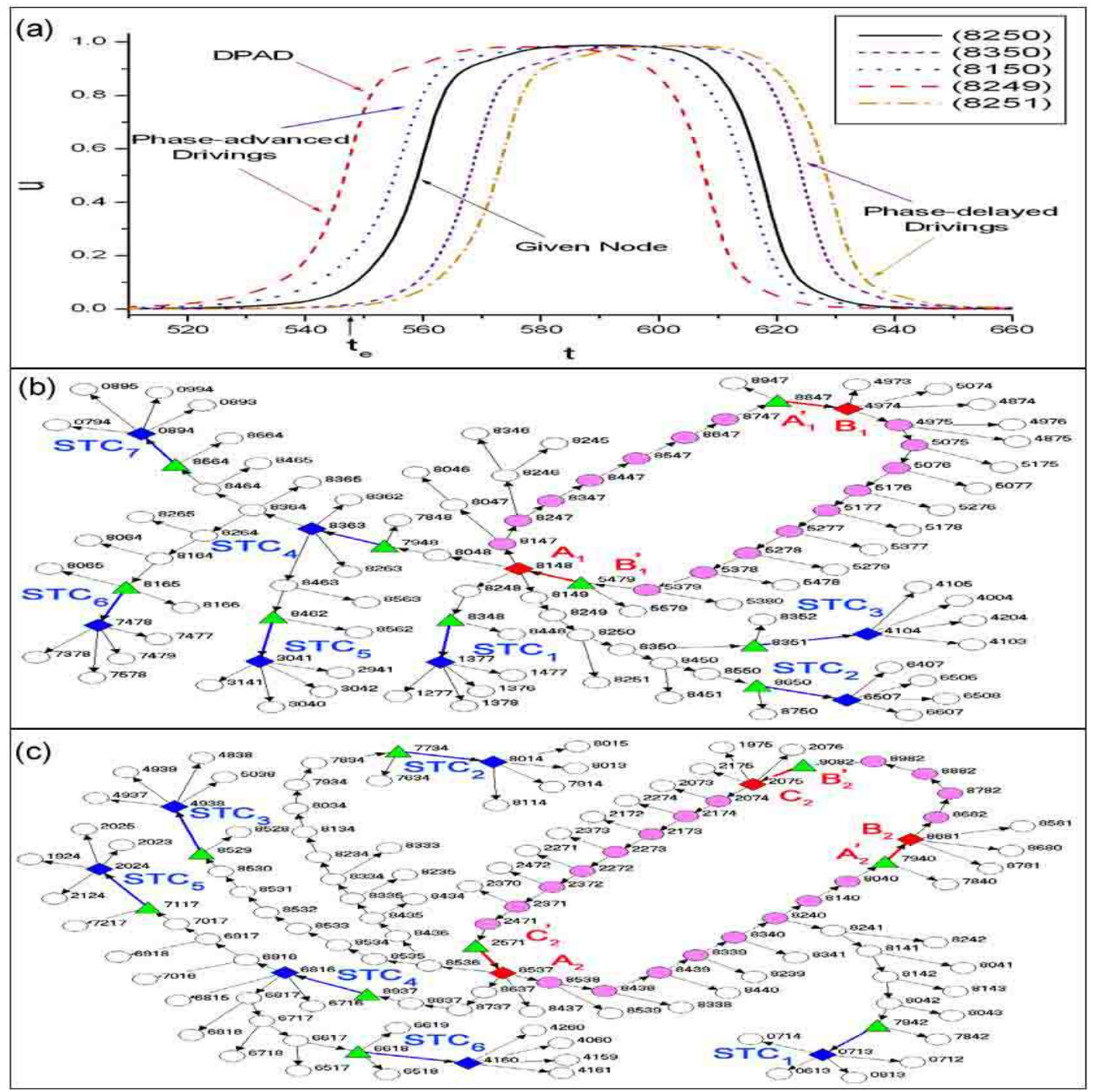

FIG. 3: Coloured online. (a) Demonstration of the phase-advanced drivings and dominant phase-advanced driving of a given node 8250 for the state Fig. 2 $\mathrm{c}$ ). Black solid curve shows the $u(t)$ signal of the given node 8250 . Other four coloured curves are the $u(t)$ signals of nodes interacting with node 8250. Jumping time $t_{e}$ is marked by the arrowed line. It is obvious that signals 8249 (red dash line) and 8150 (blue dot line) represent phase-advanced drivings, and their couplings help to excite the given node at $t_{e}$. And the node 8249 (red dash line) signal provides the most significant contribution in exciting the given node, and is identified as dominant phase-advanced driving. Signals 8350 (purple short dash line) and 8251 (yellow dash dot line) are phase-delayed interactions, and they provide no (or, precisely, play negative) contributions to kick the given node. (b) (c) TFL structures (partial nodes and DPADs are presented) corresponding to patterns Figs. 22 c) and 2 (2), respectively. All subscripts indicate the node positions, e.g., 8250 representing the node index $(i, j)$ with $i=50, j=82$. All red (arrowed to the red square source target centers) and blue (arrowed to the blue square sub-target centers) bold lines denote long-range interactions. From these TFL patterns we can identify the following information: (i) DPAD loops (linked by pink nodes) as the oscillation sources; (ii) Oscillation centers (red square nodes in source loops), i.e., $\left(A_{1}, B_{1}\right)$ for (b) and $\left(A_{2}, B_{2}, C_{2}\right)$ for (c) from which all waves are generated; (iii) Successive driving sequences representing wave propagation pathways. 


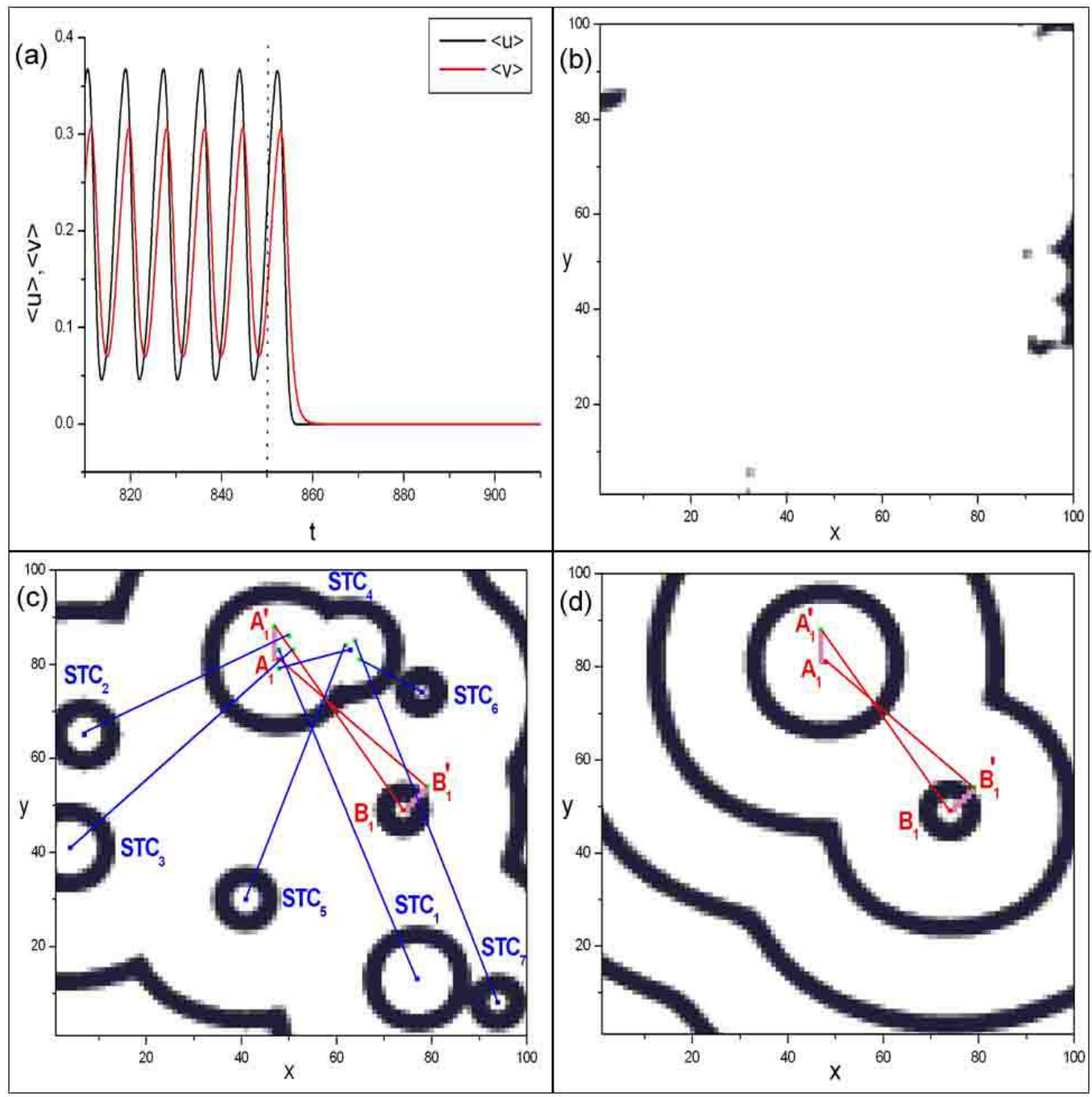

FIG. 4: Coloured online. Oscillation regulation of target waves by discarding some interacting links of Fig. 2 (c). (a) Trajectories of $<u(t)>$ $\frac{1}{N^{2}} \sum_{i, j=1}^{N=100} u_{i, j}(t)$ and $\left\langle v(t)>\frac{1}{N^{2}} \sum_{i, j=1}^{N=100} v_{i, j}(t)\right.$ with a single long-range link $B_{1}^{\prime} A_{1}$ of pattern Fig. 20 c) discarded at $t=850$ (indicated by the dot line). (b) A snapshot of pattern taken at $t=855$ evolved from pattern Fig. 2 c) after the L-RC $B_{1}^{\prime} A_{1}$ discarded. (c) (d) Snapshots of the asymptotic patterns taken at $t=1500$ with 141 long-rang links ((c)) and 148 long-rang links ((d)) discarded at $t=850$, respectively. All the remained long-range couplings are shown in (c) and (d). 


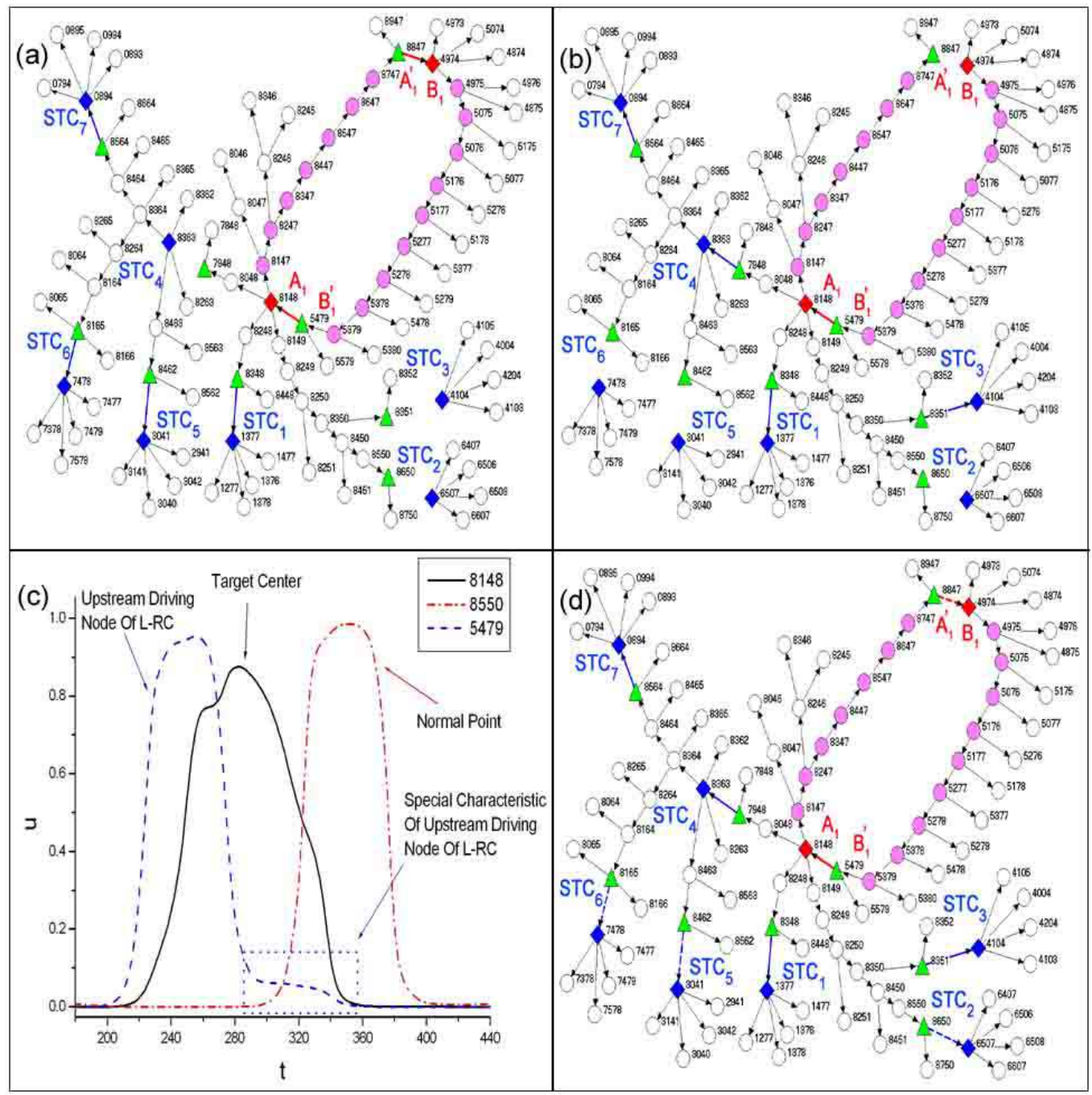

FIG. 5: Coloured online. DPAD paths of state Fig. 2 lc) with incomplete information. (a) TFL pattern with 50 L-RCs, randomly chosen, unknown. The pattern keeps the essential structure and exactly the same source loop of Fig. 3 b). (b) The same as (a) with different set of 50 L-RCs unknown. Now the source loop of Fig. 3 b) cannot be explored directly. However, this incomplete DPAD pattern is still very useful in exploring the driving structure of state Fig.2 (c). (c) Demonstration of the characteristics of periodic orbits of various nodes. Black solid, blue dash and red dash dot curves show $u(t)$ signals of a given target center 8148, a node 5479 driving the target center 8148 via a L-RC, and a node 8550 without the L-RC driving (called normal node), respectively. In (c) the L-RC driving node (blue dash curve) has the characteristic of slightly hampered tail (see the small dot frame), clearly distinguishing from the normal node (red dash dot curve with perfectly smooth tail). This distinction has been verified to be common for all nodes testified. (d) By applying the three conclusions in Sec. V and the characteristic distinctions shown in Figs. 5 ( c) we can supplement all the missing long-range driving links of the source nodes in Figs. 5 a) and 5 b) (see dot arrows in (d) for patching Fig. 5 b)). Now the TFL pattern patched from Fig. 5]b) shows exactly the same self-organized oscillation structure as Fig. 3] b). 